Original Article

\title{
INFLUENCE OF DYSLIPIDEMIA ON SOMATIC NEUROPATHY IN TYPE 2 DIABETES MELLITUS
}

\author{
Subbalakshmi N.K. ${ }^{1}$, Sathyanarayana Rao K.N. ${ }^{2}$, Adhikari P.M.R. ${ }^{3}$ \& Sheila R. Pai ${ }^{4}$ \\ ${ }^{2}$ Professor \& HoD Department of Pharmacology, K.S. Hegde Medical Academy, Mangalore \\ ${ }^{3}$ Professor, Department of Medicine, ${ }^{4}$ Professor, ${ }^{1}$ Associate Professor, Department of Physiology, Kasturba Medical \\ College, Manipal University, Mangalore - 575 001, Karnataka, India
}

Correspondence

Subbalakshmi N.K.

Associate Professor, Department of Physiology, Kasturba Medical college, Manipal University, Manglaore - 575 001, Karnataka, India Mobile : +91 94839 10879, Fax : +91 8242428183 E-mail : rao.subbalakshmink@rediffmail.com

\begin{abstract}
:
Introduction : Dyslipidemia is an established cardiovascular risk factor. But its influence on somatic neuropathy (PNP) in diabetes mellitus is not clear.

Objective of the study: This study assessed the association between lipids and PNP in diabetes mellitus.

Materials and Methods : 101 type 2 diabetics with recently done fasting lipid profile were included. Lipid profile included fasting total cholesterol, LDL-cholesterol, HDL- cholesterol and triglycerides. Based on the neurological examination diabetics were divided into two groups: with PNP $(n=53)$ and without PNP $(n=48)$. Severity of PNP was quantified based on total scoring of all the definite clinical signs of PNP. 30 healthy subjects matched for age and sex with PNP group served as control. Statistical analysis was done with suitable statistical tests. Statistical significance was taken to be at p-value less than 0.05 .
\end{abstract}

Results: Mean total cholesterol, triglycerides, LDL cholesterol of PNP group was significantly higher compared to non-diabetic control ( $p$ $<0.05 ;<0.01 ;<0.001$ respectively). There was no significant difference in any of the lipids between with PNP and without PNP groups. Among the lipids, correlation was observed only between triglycerides and PNP severity score $(r=0.37, p<0.01)$. Mean age and duration of diabetes of PNP group was significantly higher compared to without PNP group $(p<0.01 ;<0.01$ respectively).

Conclusion: Dyslipidemia persists in type 2 diabetics. Hypertriglyceridemia is correlated with severity of PNP. Higher age and duration of diabetes are associated with PNP in type 2 diabetes.

Keywords : somatic neuropathy, hypertriglyceridemia, dyslipidemia, age, duration, type 2 diabetes mellitus

\section{Introduction:}

One of the most common complications of diabetes is peripheral neuropathy, occurring approximately in $60 \%$ of all diabetic patients. ${ }^{(1,2 \text { and } 3)}$ Diabetic neuropathy is the leading cause of diabetes-related hospital admissions and non-traumatic amputations. ${ }^{(1,2 \text { and } 3)}$ Tight glycemic control has been shown to decrease the number of patients that develop micro-vascular complications; ${ }^{4}$ and ${ }^{5)}$ however, a

\begin{tabular}{|c|}
\hline Access this article online \\
\hline Quick Response Code \\
\hline
\end{tabular}
significant proportion of diabetic patients continue to develop these complications even with intensive glycemic control. (6) Additionally, in the Steno-2 study, patients treated with a multifactorial intervention including aspirin, statins, renin angiotensin blockers, glycemic control, and lifestyle modifications still developed diabetic complications at a high rate. ${ }^{(6)}$ These surprising observations are leading investigators to reconsider other potentially modifiable risk factors.

Hypertriglyceridemia has received little attention of late and is typically not considered a primary clinical target. Elevated triglycerides in concert with low HDL is associated with the metabolic syndrome and increased cardiovascular risk in healthy populations. ${ }^{(5)}$ However, the relationship between triglycerides and non-cardiovascular complications has received less attention. The EURODIAB study established a significant association between cholesterol and fasting triglycerides and the development 
of diabetic neuropathy ${ }^{(8)}$ and cardiac autonomic neuropathy. ${ }^{(9)}$ Furthermore, elevated triglycerides were recently found to correlate independently with loss of sural nerve myelin fiber density, a marker for progression of diabetic neuropathy. ${ }^{(10)}$ On the other hand, a previous study showed that there was no significant difference in serum cholesterol of type 2 diabetic patients with negative and positive sensorimotor neuropathy. ${ }^{(11)}$ Atherogenic lipid indices including total cholesterol/high-density lipoprotein cholesterol, low-density lipoprotein-cholesterol, triglycerides/high-density lipoprotein and other ratios were predictors for atherosclerosis in type 2 diabetes mellitus but not for neuropathy. ${ }^{(12,13)}$ Thus, role of dyslipidemia in pathogenesis of diabetic neuropathy is not clear.

This study was undertaken to assess the lipid profile and other associated clinical characteristics of somatic neuropathy in type 2 diabetes mellitus.

\section{Materials and Methods:}

This study was done in patients with type 2 diabetes mellitus attending the outpatient clinic at the Department of Medicine, Kasturba Medical College Hospital, Mangalore. This study was undertaken after the approval by the Institutional Ethical Committee in accordance with the Ethical Standards laid down in the Declaration of Helsinki and obtaining consent from the study participants

Study subjects: 101 consecutive type 2 diabetic patients with freshly done fasting lipid profile and fasting blood sugar were enrolled in to this study. Based on the physical examination of peripheral nervous system type 2 diabetics were divided into two groups: a. with PNP ( $n=$ 53) and b. without PNP $(n=48)$. Severity of PNP was quantified based on total scoring of all the definite clinical signs of PNP. 30 non-diabetic healthy subjects matched for age and sex with PNP group (in whom freshly done fasting lipid profile and fasting blood sugar was available) served as control.

Exclusion criteria were diabetic patients with (1) severe systemic diseases, such as liver disease and kidney failure;
(2) heart diseases in which regular sinus arrhythmia was lost; or (3) known neuropathy of other etiology.

Parameters assessed: Fasting total cholesterol, triglycerides, HDL-cholesterol, LDL-cholesterol, fasting blood sugar, blood pressure, resting heart rate, body mass index, waist - hip ratio, age and duration of diabetes mellitus.

\section{Study protocol :}

All the subjects in the study and control groups were subjected to clinical examination. However, subjects in the study group alone underwent detailed systemic examination. In the personal interviews with the patients detailed history was obtained with special reference to age, duration of diabetes mellitus, symptoms of neuropathy, diabetes-related complications and medication.

The height, weight, waist and hip circumference of all the subjects were measured. The waist circumference was measured at the midpoint between the lower margin of the last palpable rib and the top of the iliac crest. Hip circumference was measured around the widest portion of the buttocks. The measurements were taken at the end of a normal expiration while subjects in relaxed condition. The body mass index was calculated using the formula: weight in kilograms $(\mathrm{kg})$ divided by height in meters $(\mathrm{m})$ squared. Waist-hip ratio was expressed as ratio of the circumference of the waist to that of the hips.

The diverse pattern of clinical manifestations of somatic neuropathy compelled us to adopt the neurological scoring system to define somatic neuropathy. A total score of 0-3 was considered normal. A score of $\geq 4$ was considered as having somatic neuropathy. (Adopted and modified from Kennedy et al). ${ }^{(14)}$ The protocol employed is shown in Table I.

Blood pressure was measured in all the subjects. Two readings were taken five minutes apart in the sitting position. The mean of the two was recorded as blood pressure.

The resting electrocardiogram was recorded in a supine 
position using a standard electrocardiogram. Lead II electrocardiogram was then recorded continuously at a speed of $25 \mathrm{~mm} / \mathrm{s}$ for 60 seconds. The total number of R-R intervals in one minute was considered as the resting heart rate.

Statistical analysis included ANOVA, Bonferroni, Pearson correlation coefficient and unpaired t tests. Statistical significance was taken to be at $p$-value less than 0.05 .

\section{Result:}

Among the 101 type 2 diabetics 53 were diagnosed with somatic peripheral neuropathy (PNP- group) and 48 were free from somatic neuropathy (non-PNP group). We compared the lipid profile and other characteristics of study group (PNP-group) with non-diabetic healthy control and non-PNP group. We also sought the correlation between PNP severity score and clinical characteristics of PNP group.

Comparison of baseline characteristics between study group (PNP group) and non-diabetic healthy control:

Study group and the control group were comparable with regard to age and gender distribution. Body mass index of PNP group was significantly lower compared to control $(p<$ 0.01). Resting heart rate, fasting blood sugar, total cholesterol, triglycerides, LDL-cholesterol was significantly higher in PNP group compared to healthy control ( $p<$ $0.001, p<0.0001, p<0.05, p<0.01, p<0.001$ respectively, table 2 and 3 ). There was no significant difference in HDLcholesterol, blood pressure, waist /hip ratio between study and control group (table 2 and table 3 ).

Comparison of baseline characteristics between study group (PNP group) and non-PNP group:

Age and duration of diabetes of PNP group was significantly higher compared to non- PNP group ( $p<0.01$, $p<0.0001$ respectively, table 3 ). Body mass index of PNP group was significantly lower compared to non- PNP group ( $p<0.01$, table 3). There was no significant difference in waist/hip ratio, blood pressure, heart rate, total cholesterol, triglycerides, LDL, HDL and fasting blood sugar between
PNP and non-PNP groups ( Table 2 and table 3).

Correlation between PNP severity score and biochemical parameters and other associated clinical characteristics of PNP group

In the PNP group significant positive correlation was found between PNP severity score and triglycerides and duration of diabetes $(r=0.37, p<0.01$, figure $1 ; r=0.41 p<0.01$ respectively). There was no significant correlation between PNP severity score and total cholesterol, LDL cholesterol, HDL cholesterol, fasting blood sugar, systolic blood pressure, diastolic blood pressure resting heart rate and age $(r=0.23,-0.04,0.01,-0,01,-0.11,0.14,0.07,0.07$ respectively).

\section{Discussion:}

Dyslipidemia is a well-established cardiovascular risk factor. But its influence on non-cardiovascular clinical conditions is not confirmed. Thus we assessed the influence of dyslipidemia on somatic neuropathy in type 2 diabetes mellitus.

In the present study, total cholesterol, triglycerides, and LDL cholesterol was higher in PNP group compared to nondiabetic healthy control. At the same time these parameters were also significantly higher in non-PNP group compared to healthy control (Table 2). Moreover there was no significant difference in any of the measured lipids between PNP and non-PNP group (Table 2). This finding suggests that dyslipidemia persists in subjects with type 2 diabetes mellitus irrespective of presence or absence of PNP. However on the other hand, among the lipids studied, only triglycerides showed positive correlation with PNP severity score (figure 1). Thus results of our study suggest that among the lipids, mainly hypertriglyceridemia may adversely affect somatic neuropathy. Our finding is in agreement with the findings of Shanker et al who had also observed correlation between triglyceride level and severity of somatic neuropathy. ${ }^{(15)}$ But, in their study triglycerides of PNP group was higher compared to without PNP group unlike in our study. However, there are subtle differences between our study and their study in defining 
severity of PNP. We scored PNP severity based on definite signs of PNP unlike subjective symptoms of somatic neuropathy followed in the study of Shanker et al. In the study of Mac Minis PG et al in all patients with painful polyneuropathy cholesterol level was moderately increased but level of triglycerides were exceedingly high. (16) In the study of Timothy D. Wiggin et al, in subjects with mild to moderate diabetic neuropathy, elevated triglycerides correlated with sural nerve myelinated fiber density loss independent of disease duration, age, diabetes control, or other variables. ${ }^{(10)}$ Similarly in the EURODIAB cohort study, patients with type 1diabetes and elevated triglycerides were at an increased risk for the development of peripheral neuropathy in bivariate analysis. ${ }^{(8)}$ Thus it appears that hypertriglyceridemia is involved in etiopathogenesis of diabetic somatic neuropathy.

In the present study, duration of diabetes was higher in PNP group compared to non-PNP group (table 3 ). In addition, positive correlation was observed between PNP severity score and duration of diabetes. Our finding is in accordance with the findings of Dyck pJ et al and Humphries et al. ${ }^{(17 \text { and 18) }}$ who had also observed correlation between duration of diabetes and severity of PNP.

In the present study in addition, age of PNP group was higher compared to without PNP group. But there was no significant correlation between age and PNP severity score. This finding suggests that prevalence of PNP increases with aging but aging may not be a major factor in progression of PNP.

Our study is with certain limitations. Glycated hemoglobin is considered to provide an accurate and objective measure of glycaemic control over a period of weeks and months. Due to financial constraints, it was not measured. Thus associations between glycemic control and PNP could not be studied. Certain studies have implicated hypertriglyceridemia in subclinical peripheral neuropathy defined based on nerve conduction assessment. ${ }^{(19)}$ Owing to practical difficulties faced in out- patient department set-up, we could not conduct the nerve conduction studies. Thus whether, all the subjects of without PNP group were having subclinical peripheral neuropathy or not could not be confirmed.

\section{Conclusions :}

However based on our study findings it could be concluded that dyslipidemia persists in type 2 diabetics. Hypertriglyceridemia is correlated with severity of PNP. Higher age and duration of diabetes are associated with PNP in type 2 diabetes.

Table 1 : Protocol employed in systemic examination and scoring of somatic peripheral nervous system $\ddagger$

\begin{tabular}{|c|c|c|}
\hline Neurological examination & Pattern of scoring & Total score \\
\hline Muscle power* & $\begin{array}{l}\text { Grade V = } \\
\text { Grade IV = } 1 \\
\text { Grade III = } 2 \\
\text { Grade II = } \\
\text { Grade I = 4 } \\
\text { Grade } 0=5\end{array}$ & 10 \\
\hline $\begin{array}{l}\text { Reflexes: } \\
\text { Biceps } \\
\text { Quadriceps } \\
\text { Achilles }\end{array}$ & $\begin{array}{l}\text { Normal =0 } \\
\text { Sluggish = } 1 \\
\text { Absent }=2\end{array}$ & 12 \\
\hline Sensation * & $\begin{array}{l}\text { (Based on degree } \\
\text { and extension of } \\
\text { impairment) }\end{array}$ & \\
\hline $\begin{array}{l}\text { Vibration } \\
\text { Pain } \\
\text { Temperature } \\
\text { Touch }\end{array}$ & $\begin{array}{l}\text { Normal }=0 \\
\text { Impaired }=1 \\
\text { Severely impaired }=2\end{array}$ & 16 \\
\hline
\end{tabular}

$\ddagger$ adopted and modified from Kennedy et al ${ }^{[14]}$;

* Upper and lower limbs

Table 2 : Lipid profile and fasting blood sugar in study and control groups

\begin{tabular}{|c|c|c|c|}
\hline Parameters & $\begin{array}{l}\text { With PNP } \\
(n=53)\end{array}$ & $\begin{array}{l}\text { Without PNP } \\
\quad(n=48)\end{array}$ & $\begin{array}{c}\text { Non-diabetic } \\
\text { healthy } \\
\text { Control }(n=30)\end{array}$ \\
\hline $\begin{array}{l}\text { Total } \\
\text { cholesterol } \\
(\mathrm{mg} / \mathrm{dl})\end{array}$ & $215.09 \pm 46.26 \ddagger^{N S}$ & $226.62 \pm 47.88$ & $188.66 \pm 44.10$ \\
\hline $\begin{array}{l}\text { Triglycerides } \\
(\mathrm{mg} / \mathrm{dl})\end{array}$ & $192.55 \pm 125.22 t^{\mathrm{Ns}}$ & $191.00 \pm 98.17$ & $118.58 \pm 44.10$ \\
\hline $\begin{array}{l}\text { High density } \\
\text { cholesterol } \\
(\mathrm{mg} / \mathrm{dl})\end{array}$ & $45.45 \pm 12.65^{\mathrm{NS}}$ & $43.39 \pm 9.99$ & $43.04 \pm 9.21$ \\
\hline $\begin{array}{l}\text { Low density } \\
\text { cholesterol } \\
(\mathrm{mg} / \mathrm{dl})\end{array}$ & $142.94 \pm 36.86+t^{N S}$ & $150.69 \pm 36.86$ & $114.66 \pm 25.75$ \\
\hline $\begin{array}{l}\text { Fasting blood } \\
\text { sugar }(\mathrm{mg} / \mathrm{dl})\end{array}$ & $182.09 \pm 63.66^{\mathrm{NS}}$ & $171.12 \pm 66.69$ & $91.08 \pm 9.58 *$ \\
\hline
\end{tabular}


Values are mean $\pm S D, n=$ sample size, $P N P=$ somatic neuropathy.

$\ddagger p<0.05$ compared to non-diabetic healthy control

$\dagger p<0.01$ compared to non-diabetic healthy control

†† $p<0.01$ compared to non-diabetic healthy control

NS = Non-significant compared to without PNP group

$* p<0.001$ compared to with PNP and without PNP groups

Table 3 : Comparison of selected clinical characteristics of study subjects with non-diabetic healthy control and diabetic subjects without somatic neuropathy (without PNP)

\begin{tabular}{|l|c|c|c|}
\hline Parameters & Study group With PNP ( $\mathbf{n = 5 3 )}$ & Without PNP( $\mathbf{n}$ 48) & Non-diabetic healthy Control $(\mathbf{n}=\mathbf{3 0})$ \\
\hline Age (years) & $60.03 \pm 8.80^{*}$ & $52.70 \pm 9.68$ & $61.87 \pm 15.99$ \\
\hline Male /female ratio & $28 / 25$ & $25 / 23$ & $16 / 14$ \\
\hline Body mass index $\left(\mathrm{kg} / \mathrm{m}^{2}\right)$ & $22.62 \pm 2.84^{+}$ & $25.04 \pm 3.94$ & $25.2 \pm 4.16$ \\
\hline Waist hip ratio & $0.91 \pm 0.15^{\mathrm{NS}}$ & $0.93 \pm 0.06$ & $0.95 \pm 0.07$ \\
\hline Systolic blood pressure $(\mathrm{mmHg})$ & $142.30 \pm 23.43^{\mathrm{NS}}$ & $141.79 \pm 19.78$ & $136.16 \pm 17.08$ \\
\hline Diastolic blood pressure $(\mathrm{mmHg})$ & $84.16 \pm 12.10^{\mathrm{NS}}$ & $87.45 \pm 9.17$ & $80.66 \pm 6.45$ \\
\hline Resting heart rate (beats/min) & $86.78 \pm 14.69^{* *}$ & $84.64 \pm 10.70$ & $78.83 \pm 7.93$ \\
\hline Duration of diabetes (years) & $11.86 \pm 7.66 \ddagger$ & $5.02 \pm 6.06$ & - \\
\hline
\end{tabular}

Values are mean $\pm S D, n=$ sample size

$* p<0.01$ compared to without PNP group

$* * p<0.001$ compared to non-diabetic control group

$+p<0.01$ compared to without PNP group and non-diabetic healthy control

$\ddagger p<0.01$ compared to without PNP group

NS = Non-significant compared to without PNP group and non-diabetic healthy control

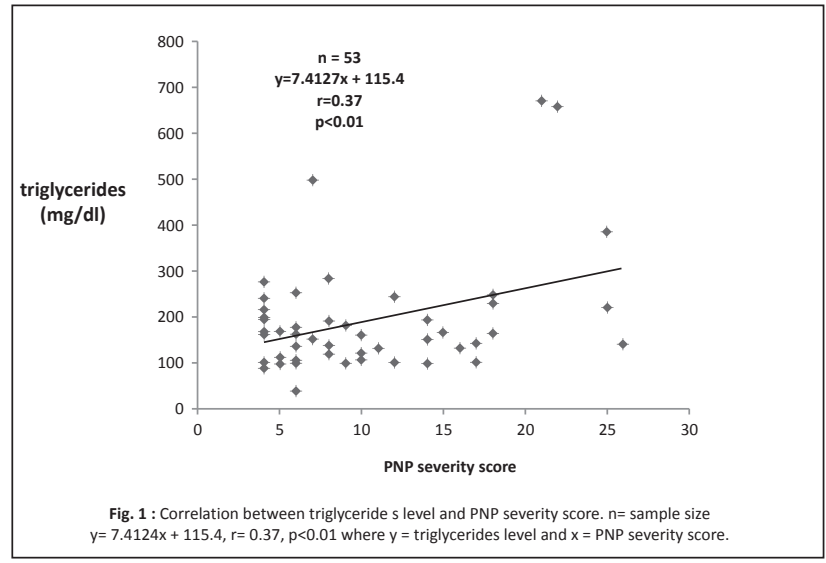

References:

1. Edwards JL, Vincent AM, Cheng HT, Feldman EL. Diabetic neuropathy: Mechanisms to management. Pharmacol Ther 2008; 120:1-34.

2. Feldman EL, Stevens MJ, Russell JW, Peltier A, Inzucchi S, Porte JD, Sherwin RS, Baron A. Somatosensory neuropathy. In The Diabetes Mellitus Manual. New York: McGraw-Hill, 2005, p. 366-384.

3. Feldman EL. Diabetic neuropathy. Curr Drug Targets 2008; 9:1-2.

4. Diabetes Control and Complications Trial Cohort. Epidemiology of Diabetes Interventions and Complications (EDIC): design, implementation, and preliminary results of a long-term follow-up of the Diabetes Control and Complications Trial Cohort. Diabetes Care 1999; 22:99-111.

5. UK Prospective Diabetes Study (UKPDS) Group. Intensive blood glucose control with sulphonylureas or insulin compared with conventional treatment and risk of complications in patients with type 2 diabetes (UKPDS 33). Lancet 1998; 352:837-853. 
neuropathy. Diabetes 2009; 58: 1634-1640.

11. Jarmuzewska EA, Ghidoni A, Mangoni AA. Hypertension and Sensorimotor Peripheral Neuropathy in Type 2 Diabetes. Eur Neurol. 2006; 57:91-5.

12. Temelkova-Kurktschiev T, Hanefeld M. The lipid triad in type 2 diabetes - prevalence and relevance of hypertriglyceridaemia/low high-density lipoprotein syndrome in type 2 diabetes. Exp Clin Endocrinol Diabetes. 2004; 112:75-9.

13. Décary S, Dumont G, Lamarche B, Hogue JC, Tremblay AJ, Bergeron J, et al. Assessment of the validity of the frequently used lipid indices for predicting LDL peak particle diameter in a large cohort of 1955 normal and dyslipidemic subjects. Clin Biochem. 2010; 43:401-6.

14. Kennedy WR, Navarrho X, Sakuta M, Mandell H, Knox CK, Sutherland $D E$. Physiological and clinical correlates of cardiorespiratory reflexes in diabetes mellitus. Diabetes Care 1989; 12; 399-408.

15. R. Shankar, Shashikiram M, Gopalraj M, Evangeline Nesa Rathnabai. Assessment of serum Triglycerides among diabetes patients with Peripheral Neuropathy. Journal of Medical Research and Practice 2012; 1(3):68-71

16. McManis PG, Windebank AJ, Kiziltan M. Neuropathy associated with hyperlipidemia. Neurology. 1994; 44(11):2185-6.

17. Dyck PJ, Davies JL, Wilson DM, Service FJ, Melton LJ 3rd, O'Brien PC: Risk factors for severity of diabetic polyneuropathy: intensive longitudinal assessment of the Rochester Diabetic Neuropathy Study cohort. Diabetes Care 199; 22:1479-1486.

18. Humphrey AR, Dowse GK, Thoma K, Zimmet PZ. Diabetes and nontraumatic lower extremity amputations. Incidence, risk factors, and prevention-a 12-year follow-up study in Nauru. Diabetes Care 1996; 19:710-714.

19. Hania S. Kassem, Sami T.Azar, Mira S. Zantout \& Raja A. Sawaya. Hypertriglyceridemia and peripheral neuropathy in neurologically asymptomatic patients. Neuroendocrinol Lett 2005; 26(6):775-779. 